\title{
ANNOUNCEMENTS
}

\section{IFSA 95}

\section{INTERNATIONAL FUZZY SYSTEMS ASSOCIATION Conference SAO PAulo, Brazil JULY 22-28, 1995}

Papers presented in the areas of

- Artificial Intelligence

- Engineering

- Mathematical Foundations
- Information Sciences

- Health Sciences, Biology, and Psychology

- Neural Networks and Hardware

- Fuzzy Systems

Area Chairs:

\section{Artificial Intelligence}

Ronald Yager

Machine Intelligence Institute Iona College

New Rochelle, NY 10801

Fax: (212) 249-1689

E-mail: rryl@iona.bitnet

\section{Engineering}

Kaoru Hirota

Dept. of Systems Control Engineering

College of Engineering

Hosei University

3-7-2 Kajino-cho

Koganey-City, Tokyo 184, Japan

Fax: 81 (423) 876241
Information Sciences

Henri Prade

Institute de Recherche en

Informatique de Toulouse

Universite de Paul Sabatier

118 Route de Narbonne

31062 Toulouse Cedex, France

Fax: 33 (61) 556239

Mathematical Foundations

Peter Klement

Fuzzy Logic Laboratorium Linz (FLLL)

Institut fuer Mathematik Johannes Kepler Universitat

A-4040 Linz, Austria

Fax: 43 (732) 246810

E-mail: klement@flll.uni-linz.ac.at
Health Sciences, Biology,
Psychology

D. Hudson

University of California, San Francisco

2615 E. Clinton Ave.

Fresno, CA 93703 USA

E-mail: hudson@medisun. UCSFresno.EDU

\section{Neural Nets and Hardware}

T. Yamakawa

Dept. of Control

Engineering \& Science

Kyushu Institute of Technology

Iizuka, Fukuoka 820, Japan

Fax: 81 (948) 297742

E-mail: yamakawa@ces.kyutech.ac.jp

\author{
Fuzzy Systems \\ J.L. Verdegay \\ Departamento Ciencias \\ de la Computacion e I.A. \\ Universidad de Granada \\ E.T.S. de Ingeneria Informatica \\ Avad. Andalucia, 38 \\ 18071 Granada, Spain \\ E-mail: jverdegay@ugr.es
}


The deadline for paper submissions has already passed; however, to attend IFSA '95, please complete the pre-registration form below and return to the Secretariat of IFSA '95 at

INPE/Setor de Eventos/IFSA '95

Av. dos Astronautos, 1758 - Caixa Postal 515

12201-970 Sao Jose dos Campos - SP - Brazil

Phone: $+55-123-418977$

Fax: +55-123-218743

E-mail: ifsa95@dep.fem.unicamp.br

\section{PRE-REgISTRATION Form \\ FOR IFSA'95}

\section{LAST Name}

First Name

Organization/Affiliation

\section{Address}

Zip/Postal Code

State

Telephone

FAx

E-mail Address
CITY

Country 


\title{
EUROPEAN CONFERENCE ON SYMBOLIC AND QUANTITATIVE APPROACHES TO REASONING AND UNCERTAINTY
}

\author{
UNIVERSITY OF FRIBOURG, SWITZERLAND \\ JULY 3-5, 1995
}

Contact:

Professor J. Kohlas

Institute of Informatics

Regina Mundi

CH-1700 Fribourg, Switzerland

Tel: +4137219560

Fax: +4137219670

E-mail: ECSQARU.iiuf@unifr.ch 